Check for updates

Cite this: J. Mater. Chem. C, 2021, 9, 13331

\title{
Supramolecular oligourethane gels as light-harvesting antennae: achieving multicolour luminescence and white-light emission through FRET $\dagger$
}

\author{
Nan Jiang, $\ddagger^{a}$ Runlin Wang, $\ddagger^{a}$ Xiaoxia You, ${ }^{a}$ Yun Geng, (D) Dongxia Zhu, (D) *a \\ Ning Zhang (D) *a and Martin R. Bryce (D) *b
}

\begin{abstract}
Soft luminescent gel materials have many practical applications due to their excellent solution processability, thixotropic, soft and facile coating performance. Here we have prepared a series of oligourethane (OU)-based gelators with fine-tuned optical properties. Chromophores with different $\pi$-conjugated lengths were introduced into the backbone in order to systematically control the energy gaps of the OUs. Blue/green/red (BGR) fluorescent gelators were thus achieved. The gelation behavior was driven by non-covalent bonds, notably hydrogen-bonds and van der Waals interactions. Because of the dynamic character of these non-covalent interactions, a simple mixture of sols could revert back to homogeneous hybrid gels. Upon gelation, the extended network of the gels leads to sequential multichromophoric Förster resonance energy transfer (FRET). Benefiting from the presence of three primary colours, the resultant hybrid gel exhibited almost any desired visible emission colour at different ratios of gelators. Of particular significance, a white-light-emitting diode (WLED) was prepared with $\mathrm{CIE}_{x, y}$ coordinates $(0.35,0.33)$ in the hybrid gel formed by precise control over the OUB/OUG/OUR stoichiometry. This type of supramolecular gel holds promise in designing large-area, flexible optical illumination, fluorescent labels and light-harvesting systems, and could be a suitable scaffold for newgeneration soft materials with tunable optical properties in display technologies.
\end{abstract}

Received 3rd July 2021,

Accepted 24th August 2021

DOI: $10.1039 / \mathrm{d} 1 \mathrm{tc} 03105 \mathrm{~h}$

rsc.li/materials-c

\section{Introduction}

Soft luminescent gel (SLG) materials that possess both solution flow characteristics and solid plasticity simultaneously, ${ }^{1}$ are desirable for the following reasons: (i) overcoming the fluid amorphous shape can effectively avoid the pollution caused by leakage, making them good for packaging. (ii) Reducing the rigidity and hardness of a pure solid improves the material's flexibility so that it becomes easy to shape, cut, deform and recover. (iii) Lowering material cost is realized due to the low concentration of the luminescent component in the SLG. Thus, SLGs have been extensively used in diverse areas like optoelectronic devices, ${ }^{2-4}$ fluorescence labels,${ }^{5-7}$ biomedicine,${ }^{8-10}$ logic

\footnotetext{
${ }^{a}$ Key Laboratory of Nanobiosensing and Nanobioanalysis at Universities of Jilin Province, Department of Chemistry, Northeast Normal University, 5268 Renmin Street, Changchun, Jilin Province 130024, P. R. China.

E-mail: zhudx047@nenu.edu.cn, zhangn380@nenu.edu.cn

${ }^{b}$ Department of Chemistry, Durham University, Durham, DH1 3LE, UK.

E-mail:m.r.bryce@durham.ac.uk

$\dagger$ Electronic supplementary information (ESI) available: Experimental details,

${ }^{1} \mathrm{H}$ NMR spectra and photophysical properties. See DOI: 10.1039/d1tc03105h

$\$$ These authors contributed equally to the preparation of this work.
}

gates, ${ }^{11}$ self-healing materials ${ }^{12}$ and micropatterning of films on flexible substrates. ${ }^{13}$ Various kinds of SLG materials, e.g., nanomaterials, ${ }^{14}$ organic-inorganic hybrid perovskite systems,${ }^{15}$ low molecular weight gelators, ${ }^{16}$ carbon dots, ${ }^{17}$ liquid crystals $^{18}$ and supramolecular gelators ${ }^{19}$ have been exploited. Compared to other kinds of gelators, supramolecular gelators can be achieved easily by physical interactions like electrostatic forces and hydrogen bonding to achieve gelation, and they can undergo reversible gel-sol-gel transitions under changed external conditions. ${ }^{20}$ Moreover, they avoid drawbacks like needing toxic heavy metals, expensive processing costs, etc. Therefore, supramolecular gelators are receiving increased attention in many fields such as intelligent materials, largescale printing manufacture and photonics. ${ }^{21-26}$ Research on supramolecular luminous gels is extensive and there remains scope for new studies on supramolecular white-light-emitting gels (WLEGs) which are of current interest. ${ }^{27-32}$ Several examples of chromophore-based gels with complementary colours for white-light-emitting soft materials were highlighted by Armaroli and co-workers in 2015. More recently, a single fluorescent polymer gel was shown by Huang et al. to aggregate via intermolecular hydrogen-bonding leading to white 
emission..$^{32}$ The polymer was obtained by copolymerization of three monomers (namely: methyl methacrylate, an acrylate derivative of ureidopyrimidone, and a vinyl derivative of a pyridinium salt of a terpyridine system) therefore, this is not an ideal or trivial process.

White-light emission can be achieved by mixing two complementary colours like turquoise and orange, or, more effectively, mixing B (blue), G (green) and R (red) colours. ${ }^{33,34}$ Generally, inter-component proximity and interaction gives rise to Förster resonance energy transfer (FRET) among shortwavelength emission (B or G) and long-wavelength emission ( $G$ or R) in these mixed systems, which leads to difficulty in tuning the emission colour. ${ }^{35,36}$ Accordingly, research has started to explore ways to avoid excess FRET and thereby control emission behaviour. Strategies include: (i) precise molecular design and careful sequential control of intercomponent interactions in either solution or solid-state, ${ }^{37}$ and (ii) partial isolation of the chromophores in a matrix to avoid redundant energy transfer. ${ }^{38}$ Both of these methods are experimentally complicated and troublesome. We recognised that supramolecular gelators could be applied in this regard, as a special kind of dispersion system, containing a lot of liquid and showing both solid and solution properties. The internal fluid should impart intrinsic spatial barriers within the gels' microstructure, offering a significant natural advantage in avoiding excess FRET and thus providing an easy way to obtain multicolour luminescence and WLEG.

Our previous work has exploited the versatility of urethane linkages to introduce different kinds of components into OU backbones to provide richer $\mathrm{H}$-bonding sites to drive the construction of gel materials with some novel optical properties, like ion sensors or mechanically-sensitive multicolour luminescent gels. ${ }^{39-41}$ Polyurethane has been claimed as the world's "sixth largest resin-based polymer group" 42 which can be regarded as an important milestone in modern technology. In recent years, oligo/polyurethanes have been designed and investigated in optical fields largely due to their advantages over many competitor materials, like large-scale production, good mechanical toughness and abrasion resistance. ${ }^{42-44}$ Moreover, there are recent promising developments in biodegradability and potential future upcycling of oligo/polyurethane materials. ${ }^{42,45}$ Therefore, oligo/polyurethane-based gelators with novel optical behaviour hold great potential for applications in everyday life. Polyurethane derivatives with white-light emission have been attempted several times in recent years, yet they are mostly PU composites with other ingredients like rareearth elements, metal ions or MOFs. ${ }^{46-48}$ An exception is in 2017, when Du et al. reported a fluorescent linear $\mathrm{CO}_{2}$-derived poly(hydroxyurethane) for cool-white LEDs (CIE 0.33, 0.38). However, the synthesis required the use of a pressurised autoclave and the polymer's emission diversity is considerably limited. ${ }^{49}$

We now report a facile light-harvesting system for multicolour luminescence and high-colour-purity white-light emission based on oligourethane (OU) gelators incorporating an organised supramolecular scaffold. (a)
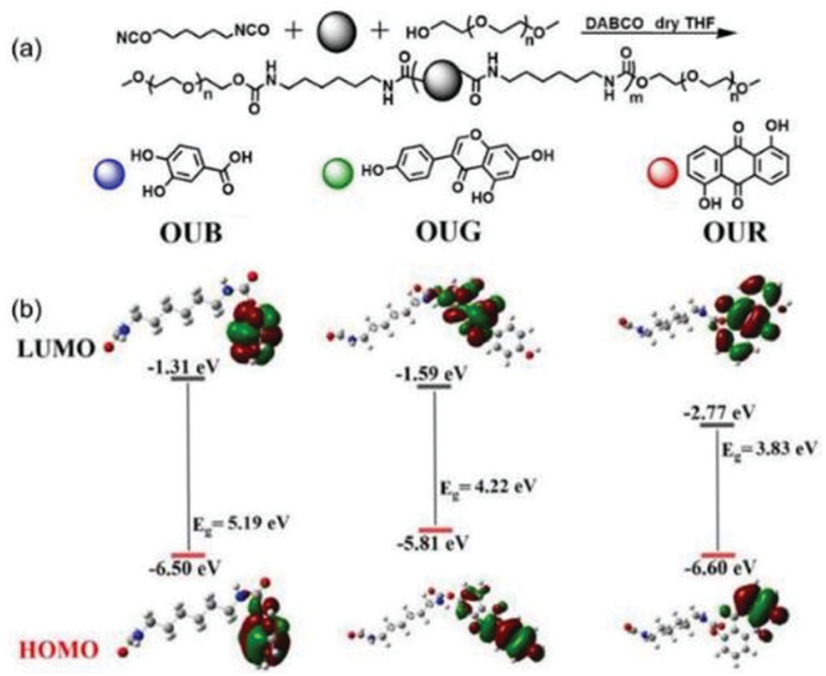

Fig. 1 (a) Synthetic routes for OUB, OUG and OUR. (b) The frontier molecular orbitals of OUB, OUG and OUR calculated at the B3LYP/6$31 \mathrm{G}(\mathrm{d}, \mathrm{p})$ level.

\section{Results and discussion}

\section{Synthesis and characterization}

The OUs shown in Fig. 1a were synthesized by reacting hexamethylene diisocyanate, polyethylene glycol monomethyl ether, the appropriate dihydroxyl ligand and 1,4-diazabicyclo[2.2.2] octane (DABCO) in anhydrous tetrahydrofuran at $75{ }^{\circ} \mathrm{C}$ for $7 \mathrm{~h}$ in a nitrogen atmosphere, whereupon the clear solution became viscous. The crude products then went through a counter precipitation process. ${ }^{1} \mathrm{H}$ NMR analysis of the final products suggested their $M_{\mathrm{n}}$ values are 2096, 2982 and $1603 \mathrm{~g} \mathrm{~mol}^{-1}$, respectively, establishing that they are oligomeric gelators (Fig. S1-S3, ESI $\dagger$ ). The OU gelators exhibited blue (OUB), green (OUG) and red (OUR) emissions, respectively. Fourier Transform infrared (FT-IR) spectroscopy showed the characteristic stretching vibrations of OUs in the solid-state (Fig. S4a, $\mathrm{ESI}+$ ). The semi-crystallinity of the OUs was shown by powder X-ray diffraction. Fig. S4b (ESI $\dagger$ ) shows the typical features of oligourethane derivatives, which offers additional physical restraints to favour the emission behaviour. ${ }^{50}$ More details of the synthesis and structural characterization are given in the ESI. $\dagger$

The rheological curve of OUB gel as a function of angular velocity is shown in Fig. S5 (ESI $\dagger$ ). At the beginning, the storage modulus $\left(G^{\prime}\right)$ is greater than the loss modulus $\left(G^{\prime \prime}\right)$, showing a typical gel phase viscoelastic region. While hydrogen bonds were gradually broken as the angular velocity increased, the system began to show prevailing viscous behavior and to flow, $G^{\prime \prime}>G^{\prime}$.

In order to prove that the gel-sol-gel process is reversible, as shown in Fig. S6a (ESI $\dagger$ ), we conducted "heating and cooling", tests. OUB gel shows specific heat-reversible characteristics of a physical gel. More importantly, when urea was added, the sol did not convert back to gel state. This is because urea can form hydrogen bonds with the amide groups, and then destroy the self-assembly of OUB molecular chains driven by hydrogen bonds. Fig. S6b (ESI $\dagger$ ) shows the concentration dependent 
emission behaviour of OUB gel within the range of 2.5 to $10 \mathrm{wt} \%$. The emission intensity increased with the increasing concentration.

OUB, OUG and OUR were obtained by controlling the $\pi$ conjugation length and energy gap $\left(E_{\mathrm{g}}\right)$. As depicted in Fig. 1b, the highest occupied molecular orbital (HOMO) and the lowest unoccupied molecular orbital (LUMO) of the gelators were calculated by density functional theory (DFT) using Gaussian program. These results demonstrate that by systematically changing the main chain structure and consequently adjusting the $E_{\mathrm{g}}$, a series of fluorescent OU gelators with BGR primary colours can be obtained. ${ }^{51}$ We selected dimethyl sulfoxide (DMSO) as the solvent for the OUs to carry out the gelation. The DMSO played two main roles: (i) it served as the external crosslinking agent, offering $\mathrm{S}=\mathrm{O} \mathrm{H}$-bonding acceptor units. (ii) Being locked into the network, occupying space, some holes formed in the micro-structure of the gels which play an important role for sequential FRET. In the process of selfassembly, solvent molecules are distributed among the chains and chromophores, giving them a defined distance from each other to form a net structure, in which the solvent molecules are subtly locked to form holes. Hence, an interconnected extended network with some holes is obtained as an efficient light-harvesting antenna allowing the multichromophore FRET process to take place within the gel system (Fig. 2a). Specifically, Fig. S7 (ESI $\dagger$ ) shows the normalized absorbance and emission spectra of OUB, OUG and OUR gels. Their emission covers the entire visible region, which offers an opportunity for a BGR colour adjustment strategy. Besides, there are some overlap areas between OUB-UV and OUG-PL/OUR-PL, OUG-UV and OUR-PL, indicating the possibility of FRET. Accordingly, OUB/OUG will act as donor molecules and OUG/OUR will act as acceptor molecules. The special structure of gels will reduce the distance between these OU units, inducing emission and donor-acceptor orbital energy overlaps to promote sequential FRET. Thereby tuneable luminescence colour is achieved which is particularly important for white-light-emitting behaviour (Fig. 2b). Taking advantage of appropriate FRET in the gel state, by precise control over the OUB/OUG/OUR stoichiometry a supramolecular white light-emitting gel (WLEG) was successfully obtained with Commission Internationale d'Eclairage (CIE) coordinates $(0.35,0.33)$ indicating high colour purity close to the ideal white light $(0.33,0.33) .{ }^{34}$ Also importantly, almost any chosen homogeneous hybrid fluorescent gel could be obtained by tailoring the ratios of the constituent OUs due to their near-identical self-assembly environments and morphology. Such a property is clearly beneficial for the solid-state lighting and large-area display applications in the future.

\section{Self-assembly behavior and gelation}

All the OUs are good supramolecular gelators that can selfassemble through $\mathrm{H}$-bonds and electrostatic interactions. The $\mathrm{H}$-bonds affect the emitting behaviour of the OUs. In the solid and gel states, closer packing results in a red-shift of the fluorescence along with the emergence of multiple peaks caused by different aggregation modes, leading to a variety of

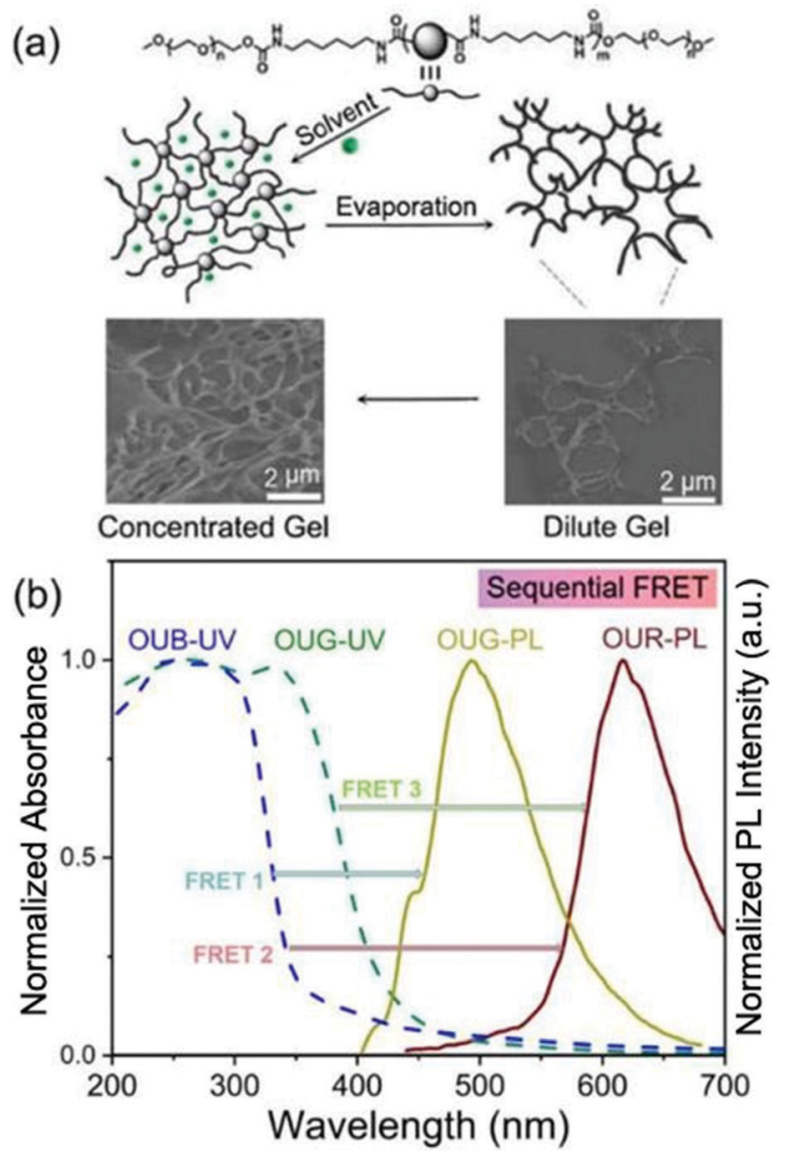

Fig. 2 (a) Proposed model for gelation process of OUs and TEM images of dilute and concentrated gel. (b) UV-vis absorption and PL emission spectra of the OUs in the gel state and illustration of FRET among OUB, OUG and OUR gels.

emitting species (Fig. S8a-c and Table S1, ESI $\dagger$ ). ${ }^{52}$ The emission lifetimes are consistent with fluorescence, not phosphorescence (Table S1, ESI $\dagger$ ). For a deeper understanding of the changeable emission behaviour, FT-IR spectra were obtained to investigate the effects of inter/intramolecular $\mathrm{H}$-bonds on the self-assembly process of the OUs (Fig. S9, ESI $\dagger$ ). The peaks at $1752 \mathrm{~cm}^{-1}$ to $1500 \mathrm{~cm}^{-1}$ are from different $\mathrm{C}=\mathrm{O}$ stretching vibrations (free $\mathrm{C}=\mathrm{O}$ and/or $\mathrm{H}$-bonded $\mathrm{C}=\mathrm{O}$ groups) in the $\mathrm{OU}$ skeletons. Fig. S9a (ESI $\dagger$ ) shows that in dilute THF solution, the OUB chains are separated by THF molecules at significant distances, so that only a limited number of hydrogen bonds are formed, and most of the rest is free $\mathrm{C}=\mathrm{O}$. In contrast, in DMSO solution, the content of free $\mathrm{C}=\mathrm{O}$ disappeared, which is associated with the DMSO molecules offering more H-bonding sites, compared to THF.

In the solid-state, for $\mathbf{O U B}$, some of the $\mathrm{C}=\mathrm{O}$ groups are not involved in H-bonding (Fig. S9a, ESI $\dagger$ ). Whereas, in the gel state, tighter cross-linking ensures all $\mathrm{C}=\mathrm{O}$ groups participate in enhancing intermolecular van der Waals and H-bonding interactions. OUG and OUR showed very similar features (Fig. S9b and c, ESI $\dagger$ ) establishing that different interactions affect the gel process and thus affect emission behaviour. 

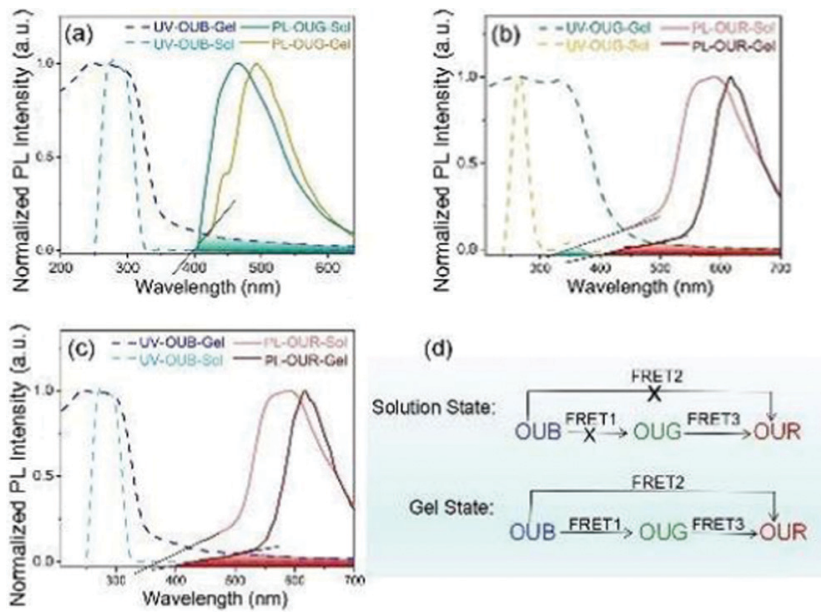

Fig. 3 Normalized absorption and emission spectra of OUs in sol and gel states. (a) FRET between OUB and OUG. (b) FRET between OUG and OUR (c) FRET between OUB and OUR (d) Schematic representation of the FRET processes in a mixture of OUB, OUG, and OUR in sol and gel states.

The morphology of the OU gels was characterized by environmental Scanning Electron Microscopy (SEM). The SEM images of OUB in DMSO clearly revealed a homogeneous net structure with holes. The morphology is concentrationdependent, with an extended polymeric network structure formed with increasing OUB concentration (Fig. S10, ESI $\dagger$ ). Besides, OUG, OUR and their mixed gels show the same homogeneous net morphology at the micrometer level (Fig. S11, ESI $\dagger$ ). This structure is surprising, as described below.

An efficient FRET among chromophores requires specific conditions to be fulfilled including spectral overlap and the distance among chromophores (1-10 nm), etc. ${ }^{36,53}$ The optical properties of the hybrid gels were investigated using absorption and emission spectroscopy measurements. Fig. 3 shows the overlap of the UV-vis spectra of OUs with their emission spectra in the gel and solution states, respectively. In Fig. 3a and Fig. S12a (ESI $\dagger$ ), OUB exhibits a narrow absorption peak at $276 \mathrm{~nm}$, which corresponds to the conjugated system of $\mathrm{O}$ and/ or $\mathrm{N}$ atoms with the aromatic rings. ${ }^{54}$ Whereas in the gel state, the absorption maximum of OUB is broadened to $c a .65 \mathrm{~nm}$, over 236-301 nm. OUG and OUR also show the analogous broadening in absorption of the gel state (Fig. S12, ESI $\dagger$ ). From Fig. S10 and S11 (ESI $\dagger$ ) discussed above, the microstructure indicated that the extended network structure may be responsible for the broadened absorption peaks. More entangled and aggregated chains in the gel state can be responsible for such spectral broadening and shift, rooted in the increased distance among chains and the number of $\mathrm{H}$-bond receptors compared to the solution state.

\section{FRET process in solution state}

To check whether a FRET process is possible between OUB and OUG, we compared the absorption spectrum of OUB and emission spectrum of OUG in Fig. 3a, and there is no significant overlap. To make sure, a stock OUB solution $\left(10^{-3} \mathrm{M}\right)$ was prepared and then OUG solution $\left(10^{-5} \mathrm{M}\right)$ was gradually added. There was a small decrease in emission intensity of OUB, but no increase in the emission intensity of OUG at an excitation of $365 \mathrm{~nm}$ (Fig. S13a, ESI $\dagger$ ). Thus, in the solution state, no FRET process is expected from OUB to OUG. Similarly, the absorption spectrum of OUB and emission spectrum of OUR in Fig. $3 c$ have no overlap. Also, when gradually adding OUR solution $\left(10^{-5} \mathrm{M}\right)$ to OUB solution $\left(10^{-3} \mathrm{M}\right)$, there was no increase in the emission intensity of OUR at an excitation of $365 \mathrm{~nm}$ (Fig. S13b, ESI $\dagger$ ). Therefore, in the solution state, there is no FRET from OUB to OUG and from OUB to OUR. Fig. 3b clearly shows a small spectral overlap between the absorption spectrum of OUG and the emission spectrum of OUR in the solution states, implying the presence of limited FRET from OUG to OUR in solution. Therefore, we prepared OUG solution $\left(10^{-3} \mathrm{M}\right)$ and added OUR solution $\left(10^{-5} \mathrm{M}\right)$ gradually when excited at $365 \mathrm{~nm}$ (Fig. S13c, ESI $\dagger$ ). The spectra show a decrease of emission intensity of OUG, whereas the emission intensity of OUR increased, suggesting there is a FRET process from OUG to OUR in solution. Hence, in the mixture of OUB, OUG and OUR solutions at an excitation of $365 \mathrm{~nm}$, FRET occurs only from OUG to OUR.

\section{FRET process in gel state}

In the gel state, intermolecular distances are decreased compared to solution state, flexible chains entangle and extend with each other, facilitating an appreciable energy transfer. Returning to Fig. 3a and c, we compared the UV-vis absorption spectrum of OUB and the emission spectra of OUG and OUR. There is no overlap in solution state but there is excellent spectral overlap between the absorption spectrum of OUB and the emission spectra of OUG or OUR in the gel state, suggesting that from OUB to OUG and from OUB to OUR energy transfer occurs only in the gel state. To get a more accurate conclusion, we blended OUB gel ( $c=4$ wt $\%)$ with $10 \%$ OUR gel ( $c=4$ wt $\%)$ : the emission intensity of OUB gel decreased and the emission intensity of OUR gel increased (Fig. S13d, ESI $\dagger$ ).

This result suggests that in the gel state, FRET occurs from OUB to OUR. Fig. S13e (ESI $\dagger$ ) clearly shows that when gradually adding OUR solution $\left(10^{-3} \mathrm{M}\right)$ to the mixture of OUB gel $(c=$ $4 \mathrm{wt} \%)$ and $2 \%$ OUG gel $(c=4 \mathrm{wt} \%)$ there is an incremental decrease of emission intensity of OUB and OUG gel, while the emission intensity of OUR steadily increases, indicating FRET process from OUB to OUG and then to OUR in the gel state. Therefore, in the gel state, the FRET occurs from OUB to OUG, from OUB to OUR and from OUG to OUR.

To reiterate the conclusions from the above results, the FRET process can be summarized as follows. In the gel state, FRET occurs from OUB to OUG, from OUG to OUR, and from OUB to OUR; however, in the solution state, FRET occurs only from OUG to OUR (Fig. 3d). Thus, in the gel state, a combination of hybrid gels in a suitable ratio can produce white-light emission.

For a better understanding of the energy transfer process among the OUs, as shown in Table $\mathrm{S} 2$ in ESI, $\dagger$ the recombination energy $\lambda(\mathrm{eV})$, Gibbs free energy difference $\Delta G(\mathrm{eV})$, energy transfer rate $k_{\mathrm{ET}}$ of the FRET process were simulated by 
Gaussian program at specific molar ratios of the OUs. The energy transfer rates of $1.88 \times 10^{13}, 5.03 \times 10^{13}$ and $2.23 \times 10^{14}$, respectively, indicated efficient and fast FRET between OUG-OUR, OUB-OUG and OUB-OUR. ${ }^{55,56}$

\section{Solution processability and application}

The tunability of the emission colour and shape of soft gel materials has received wide attention. ${ }^{57,58}$ Besides, the excellent optical processability and viscoelastic properties of our new OUGs prompted us to exploit them for coating various substrates. Since these gels are formed by weak non-covalent interactions, upon heating or other external stimuli, they were readily transformed from gels to fluid sols, and this process is reversible. The emission colour of hybrid gels was easily adjusted by changing the types and/or proportion of OUs based on the natural dynamic character of these non-covalent bonds and exploiting the principle of three primary colours. Gels were prepared with components mixed in different ratios, followed by heating and ultrasonic processing to convert the mixed gels to sols and to ensure thorough mixing, followed by cooling to room temperature to reproduce hybrid gels. Nine luminescent colours including blue, orange-red, violet, yolk yellow, white, green and cyan were obtained (Fig. 4). The OU gels were coated onto a UV torch: by varying the components of the OU gels multicolour emissions were observed under $365 \mathrm{~nm}$ light excitation. Indeed, almost any desired emission colour could be obtained by such a simple operation (Fig. S14, ESI $\dagger$ ). OUB retains $c a .25 \%$ emission intensity after continuous ultraviolet irradiation for 5 days as shown in Fig. S13f (ESI $\dagger$ ). The selected solvent (DMSO) with high boiling point is beneficial for the photostability.

To further demonstrate the potential of OUs for practical luminous applications, a white-light-emitting diode (WLED) was easily fabricated. The viscous hybrid gel was deposited onto the surface of a commercial blue LED to form a homogeneous coating. Upon turning on the LED white-light with high colour purity and CIE coordinates $(0.35,0.33)$ was

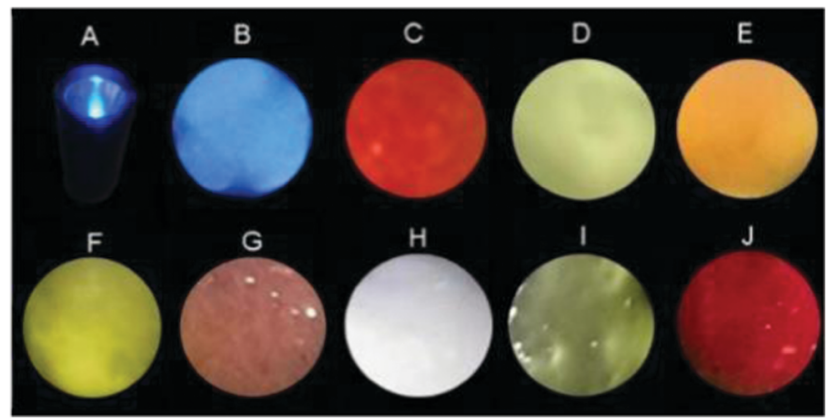

Fig. 4 A photograph of OU gels coated on a UV torch under sunlight. The emission of $\mathrm{OU}$ gels coated on the torch with different types and ratios (A) torch with no gel, (B) pure OUB, (C) OUB/OUR of $3: 1$, (D) OUB/OUG of $3: 4$, (E) OUG/OUR of $4: 5$, (F) OUB/OUR of $12.5: 1$ (G) OUB/OUR of $4: 3$, (H) OUB/OUG/OUR of $36: 1: 2.1$, (I) OUB/OUG/OUR of $7: 0.25: 0.1$, (J) OUG/OUR of $1: 3$ after the torch was lit. The excitation wavelength is $365 \mathrm{~nm}$. The ratio is the mass ratio.
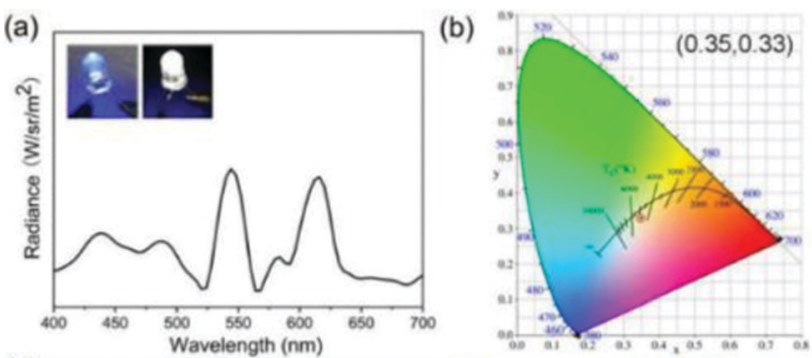

(c)

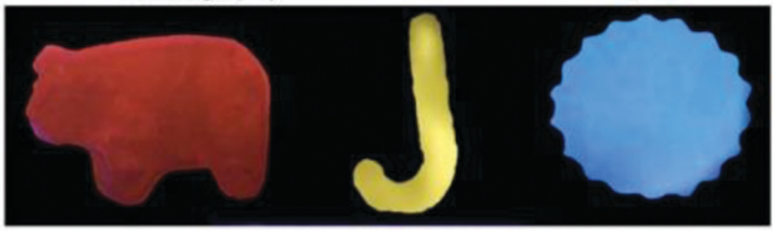

Fig. 5 (a) Emission spectra of an illuminating ultraviolet LED with molar ratio OUB: OUG: OUR of 14.7:1:1 upon excitation at $365 \mathrm{~nm}$. Photographs of the same LED coated with OUs under daylight and showing white-light after switching on the LED. (b) CIE chromaticity diagram of the white-light emission in (a). (c) Images of colour/shape-on-demand objects obtained using the hybrid gels after removing the hollow mould.

observed (Fig. 5a and b). This result clearly demonstrates that due to the partial energy transfer from OUB to OUG and to OUR, and from OUG to OUR, the fluorescence spectrum is very broad (400-700 nm). The spectrum contains three sectors of characteristic peaks from OUB, OUG and OUR, yielding whitelight emission. These hybrid gels possess excellent stability, flexibility and versatility, and could therefore be applied for (colour/shape)-on-demand multifunctional materials. To prove this concept, gels were poured into different hollow moulds. After removing the moulds, the OU gels retained the unique and versatile shapes of the moulds (Fig. $5 \mathrm{c}$ ).

\section{Conclusions}

In this work, taking advantage of the excellent solution processability, thixotropic, soft and facile coating performance of oligourethane gelators, we obtained supramolecular gels with colour-tunable and white-light emission with potential applications in WLEDs and other large-area coating and/or adhesive and display fields. BGR fluorescent gelators were achieved from simple one-pot reactions by controlling the conjugation length and energy gap of oligourethane chains. Non-covalent processes like hydrogen-bonds, electrostatic interactions and van der Waals interactions are used as the driving force to complete the gelation process. Facile mixtures formulated from two or three kinds of sols could be converted back to homogeneous hybrid gels due to the dynamic character of these non-covalent driving forces. Upon gelation, more entangled and aggregated chains resulted in extended net structures. The appropriate interchromophore distance with holes within the extended structure was formed by the incorporated solvent. FRET was successfully achieved for the hybrid gels due to the spectral overlap and distance among chromophores. Hence, any desired emission colour could be obtained from the homogeneous 
hybrid gels based on the three primary colour elements, notably including white-light emission by tailoring the types and proportions of the OUs. Such gels with highly controllable excellent optical and viscoelastic properties can undergo reversible gel-sol-gel behaviour, and they show promise in many colourful applications such as the manufacture of flexible devices, biological sciences and portable sensing kits. They may also contribute to the next generation intelligent materials by combining these properties with printable and biodegradable electronic technology. ${ }^{59}$

\section{Conflicts of interest}

There are no conflicts to declare.

\section{Acknowledgements}

This work was funded by NSFC (No. 52073045), the Key Scientific and Technological Project of Jilin Province (20190701010GH), and the Development and Reform Commission of Jilin Province (2020C035-5). D. Z. is grateful for the support from the Key Laboratory of Nanobiosensing and Nanobioanalysis at the Universities of Jilin Province. The authors acknowledge the support from the Jilin Provincial Department of Education. M. R. B. thanks EPSRC grant EL/L02621X/1 for funding.

\section{Notes and references}

1 S. Bhattacharya and S. K. Samanta, Chem. Rev., 2016, 116, 11967-12028.

2 P. Sutar, V. M. Suresh and T. K. Maji, Chem. Commun., 2015, 51, 9876-9879.

3 Q. Lin, B. Sun, Q.-P. Yang, Y.-P. Fu, X. Zhu, Y.-M. Zhang and T.-B. Wei, Chem. Commun., 2014, 50, 10669-10671.

4 Y. Xu, J. Chen, H. Zhang, H. Wei, L. Zhou, Z. Wang, Y. Pan, X. Su, A. Zhang and J. Fu, J. Mater. Chem. C, 2020, 8, 247-252.

5 H. Zhao, C. Liu, Z. Gu, L. Dong, F. Li, C. Yao and D. Yang, Nano Lett., 2020, 20, 252-260.

6 L.-J. Chen, G.-Z. Zhao, B. Jiang, B. Sun, M. Wang, L. Xu, J. He, Z. Abliz, H. Tan, X. Li and H.-B. Yang, J. Am. Chem. Soc., 2014, 136, 5993-6001.

7 R. Li, S. Wang, Q. Li, H. Lan, S. Xiao, Y. Li, R. Tan and T. Yi, Dyes Pigm., 2017, 137, 111-116.

8 C. Kim, K. Y. Kim, J. H. Lee, J. Ahn, K. Sakurai and S. S. Lee, ACS Appl. Mater. Interfaces, 2017, 9, 3799-3807.

9 B. Xie, R. L. Parkhill, W. L. Warren and J. E. Smay, Adv. Funct. Mater., 2006, 16, 1685-1693.

10 X. Cheng, Y. Jin, T. Sun, R. Qi, H. Li and W. Fan, Colloids Surf., B, 2016, 141, 44-52.

11 M. Ikeda, T. Tanida, T. Yoshii, K. Kurotani, S. Onogi, K. Urayama and I. Hamachi, Nat. Chem., 2014, 6, 511-518.

12 M. Zhang, D. Xu, X. Yan, J. Chen, S. Dong, B. Zheng and F. Huang, Angew. Chem., Int. Ed., 2012, 51, 7011-7015.
13 J. Lai, H. Zhou, Z. Jin, S. Li, H. Liu, X. Jin, C. Luo, A. Ma and W. Chen, ACS Appl. Mater. Interfaces, 2019, 11, 26412-26420.

14 Y. Lei, Q. Liao, H. Fu and J. Yao, J. Am. Chem. Soc., 2010, 132, 1742-1743.

15 B. Jeong, I. Hwang, S. H. Cho, E. H. Kim, S. Cha, J. Lee, H. S. Kang, S. M. Cho and H. Choi, ACS Nano, 2016, 10, 9026-9035.

16 V. M. Suresh, A. De and T. K. Maji, Chem. Commun., 2015, 51, 14678-14681.

17 Y. Li, M. J. Männel, N. Hauck, H. P. Patel, G. K. Auernhammer, S. Chae, A. Fery, J. Li and J. Thiele, Angew. Chem., Int. Ed., 2021, 60, 6724-6732.

18 Y.-J. Choi, W.-J. Yoon, D.-Y. Kim, M. Park, Y. Lee, D. Jung, J.-S. Kim, Y.-T. Yu, C.-R. Leeb and K.-U. Jeong, Polym. Chem., 2017, 8, 1888-1894.

19 R. Li, W. Chen, Y. Yang, H. Li, F. Xu, Z. Duan, T. Liang, H. Wen and W. Tian, Polym. Chem., 2020, 11, 5642-5648.

20 D. B. Amabilino, D. K. Smith and J. W. Steed, Chem. Soc. Rev., 2017, 46, 2402-2420.

21 F. Gu, C. Zhang and X. Ma, Macromol. Rapid Commun., 2019, 40, 1800751.

22 H.-J. Kim, H. J. Lee, J. W. Chung, D. R. Whang and S. Y. Park, Adv. Opt. Mater., 2018, 7, 1801348.

23 Z. Tang, X. Lyu, L. Luo, Z. Shen and X.-H. Fan, ACS Appl. Mater. Interfaces, 2020, 12, 45420-45428.

24 P. Bairi, B. Roy, P. Chakraborty and A. K. Nandi, ACS Appl. Mater. Interfaces, 2013, 5, 5478-5485.

25 D. K. Maiti and A. Banerjee, Chem. Commun., 2013, 49, 6909-6911.

26 Q. Zhu, L. Zhang, K. V. Vliet, A. Miserez and N. Holten-Andersen, ACS Appl. Mater. Interfaces, 2018, 10, 10409-10418.

27 C. Chen, J. Chen, T. Wang and M. Liu, ACS Appl. Mater. Interfaces, 2016, 8, 30608-30615.

28 L. Yang, J. Huang, M. Qin, X. Ma, X. Dou and C. Feng, Nanoscale, 2020, 12, 6233-6238.

29 V. K. Praveen, C. Ranjith and N. Armaroli, Angew. Chem., Int. Ed., 2014, 53, 365-368.

30 S. Das, N. Okamura, S. Yagi and A. Ajayaghosh, J. Am. Chem. Soc., 2019, 141, 5635-5639.

31 T. S. Mahapatra, H. Singh, A. Maity, A. Dey, S. K. Pramanik, E. Suresh and A. Das, J. Mater. Chem. C, 2018, 6, 9756-9766.

32 H. Wang, X. Ji, Z. Li, C. N. Zhu, X. Yang, T. Li, Z. L. Wu and F. Huang, Mater. Chem. Front., 2017, 1, 167-171.

33 C. Vijayakumar, V. K. Praveen and A. Ajayaghosh, Adv. Mater., 2009, 21, 2059-2063.

34 V. Anand, R. Mishra and Y. Barot, Dyes Pigm., 2021, 191, 109390. 35 D. K. Maiti, R. Bhattacharjee, A. Datta and A. Banerjee, J. Phys. Chem. C, 2013, 117, 23178-23189.

36 S. Jiang, M. D. Newton and R. J. Silby, Phys. Rev. Lett., 2004, 92, 218301.

37 Z. Xie, C. Chen, S. Xu, J. Li, Y. Zhang, S. Liu, J. Xu and Z. Chi, Angew. Chem., Int. Ed., 2015, 54, 7181-7184.

38 C. Kim, Y. J. Gwon, J. Kim and T. S. Lee, Polym. Chem., 2018, 9, 5671-5679.

39 N. Jiang, S.-H. Ruan, X.-M. Liu, D. Zhu, B. Li and M. R. Bryce, Chem. Mater., 2020, 32, 5776-5784. 
40 N. Jiang, D. Zhu, Z. Su and M. R. Bryce, J. Mater. Chem. C, 2020, 8, 5137-5142.

41 Y. Feng, N. Jiang, D. Zhu, Z. Su and M. R. Bryce, J. Mater. Chem. C, 2020, 8, 11540-11545.

42 R. N. C. Utomo, W.-J. Li, T. Tiso, C. Eberlein, M. Doeker, H. J. Heipieper, A. Jupke, N. Wierckx and L. M. Blank, ACS Sustainable Chem. Eng., 2020, 8, 17466-17474.

43 A. Lendlein and S. Kelch, Angew. Chem., Int. Ed., 2002, 41, 2034-2057.

44 D. K. Chattopadhyay and K. V. S. N. Raju, Prog. Polym. Sci., 2007, 32, 352-418.

45 M. J. C. Espinosa, A. C. Blanco, T. Schmidgall, A. K. AtanasoffKardjalieff, U. Kappelmeyer, D. Tischler, D. H. Pieper, H. J. Heipieper and C. Eberlein, Front. Microbiol., 2020, 11, 404.

46 M. B. Simões, S. Ullah, C. Hazra, M. W. C. Man, S. J. L. Ribeiro and U. P. Rodrigues-Filho, J. Lumin., 2018, 201, 384-389.

47 Y. Jin, M. Shi, Y. Zhu, Z. Pang, X. Li and M. Ge, Mater. Res. Express, 2021, 8, 025701.

48 Y. Tang, W. Cao, L. Yao, Y. Cui, Y. Yu and G. Qian, J. Mater. Chem. C, 2020, 8, 12308-12313.

49 B. Liu, Y.-L. Wang, W. Bai, J.-T. Xu, Z.-K. Xu, K. Yang, Y.-Z. Yang, X.-H. Zhang and B.-Y. Du, J. Mater. Chem. C, 2017, 5, 4892-4898.

50 Q. Zhou, B. Cao, C. Zhu, S. Xu, Y. Gong, W. Z. Yuan and Y. Zhang, Small, 2016, 12, 6586-6592.
51 X. Zhao, M. R. Pinto, L. M. Hardison, J. Mwaura, J. Müller, H. Jiang, D. Witker, V. D. Kleiman, J. R. Reynolds and K. S. Schanze, Macromolecules, 2006, 39, 6355-6366.

52 N. Jiang, G.-F. Li, B.-H. Zhang, D.-X. Zhu, Z.-M. Su and M. R. Bryce, Macromolecules, 2018, 51, 4178-4184.

53 E. Hirata1 and E. Kiyokawa, Biophys. J., 2016, 111, 1103-1111.

54 Q. Wan, M. Liu, L. Mao, R. Jiang, D. Xu, H. Huang, Y. Dai, F. Deng, X. Zhang and Y. Wei, Mater. Sci. Eng., C, 2017, 72, 352-358.

55 B. S. B. Karunathilaka, U. Balijapalli, C. A. M. Senevirathne, Y. Esaki, K. Goushi, T. Matsushima, A. S. D. Sandanayaka and C. Adachi, Adv. Funct. Mater., 2020, 30, 2001078.

56 W. Li, Q. Huang, Z. Yang, X. Zhang, D. Ma, J. Zhao, C. Xu, Z. Mao, Y. Zhang and Z. Chi, Angew. Chem., Int. Ed., 2020, 59, 22645-22651.

57 K.-J. Ko, H. B. Lee, H. M. Kim, G. J. Lee, S.-R. Shin, N. Kumar, Y. M. Song and J.-W. Kang, Nanoscale, 2018, 10, 16184-16192.

58 J. B. Prasannakumar, Y. S. Vidya, K. S. Anantharaju, G. Ramgopal, H. Nagabhushana, S. C. Sharma, B. Daruka Prasad, S. C. Prashantha, R. B. Basavaraj, H. Rajanaik, K. Lingaraju, K. R. Prabhakara and H. P. Nagaswarupa, Spectrochim. Acta A, 2015, 151, 131-140.

59 K. Nyamayaro, P. Keyvani, F. D’Acierno, J. Poisson, Z. M. Hudson, C. A. Michal, J. D. W. Madden, S. G. Hatzikiriakos and P. Mehrkhodavandi, ACS Appl. Mater. Interfaces, 2020, 12, 52182-52191. 